\title{
Multivoltage Floorplan Design
}

\author{
Qiang Ma and Evangeline F. Y. Young
}

\begin{abstract}
Energy efficiency has become a very important issue to be addressed in today's system-on-a-chip (SoC) designs. One way to lower power consumption is to reduce the supply voltage. Multisupply voltage (MSV) is thus introduced to provide flexibility in controlling the power and performance tradeoff. In region-based MSV, circuits are partitioned into "voltage islands" where each island occupies a contiguous physical space and operates at one voltage level. These tasks of island partitioning and voltage level assignment should be done simultaneously in the floorplanning process in order to take those important physical information into consideration. In this paper, we consider this core-based voltage island driven floorplanning problem including islands with power down mode, and propose a method to solve it. Given a candidate floorplan solution represented by a normalized Polish expression, we are able to obtain optimal voltage assignment and island partitioning (including islands with power down mode) simultaneously to minimize the total power consumption. Simulated annealing is used as the basic searching engine. By using this approach, we can achieve significant power saving (up to $50 \%$ ) for all datasets, without any significant increase in area and wire length. We compared our approach with the most updated previous work on the same problem, and results show that our approach is much more efficient and is able to save more power in most cases. We have also studied two other approaches to solve the same problem, a simple dynamic programming approach and a lowest possible power consumption approach. Experimental results show that ours can perform the best among these three approaches. Our floorplanner can also be extended to minimize the number of level shifters, to address a minVdd version of the problem and to simplify the power routing step by placing islands close to their corresponding power pins.
\end{abstract}

Index Terms-Floorplanning, low power, voltage island, voltage scaling.

\section{INTRODUCTION}

$\mathbf{T}$ HERE ARE two kinds of power consumption: dynamic and leakage. Dynamic power is caused by charging and discharging of the load capacitance during switching, while leakage power is due to subthreshold currents when a device is turned off. Energy efficiency is now an important issue

Manuscript received June 16, 2009; revised October 11, 2009. Current version published March 19, 2010. This work was partially supported by a grant from the Research Grants Council of the Hong Kong Special Administrative Region, China (Project No. 4184/07). The preliminary version of this article appeared in the Proceedings of the 2007 International Conference on Computer-Aided Design [6]. This paper was recommended by Associate Editor, I. Bahar.

Q. Ma is with the Department of Electrical and Computer Engineering, University of Illinois at Urbana-Champaign, Champaign, IL 61821 USA (e-mail: qiangma1@illinois.edu).

E. F. Y. Young is with the Department of Computer Science and Engineering, Chinese University of Hong Kong, Hong Kong, China (e-mail: fyyoung@cse.cuhk.edu.hk).

Color versions of one or more of the figures in this paper are available online at http://ieeexplore.ieee.org.

Digital Object Identifier 10.1109/TCAD.2010.2042895 in today's system-on-a-chip (SoC) designs because of the increasing power density and the popularity of portable systems. There are many techniques to reduce power consumption. One of the most effective ways is by lowering the voltage supply. Multivoltage design is thus introduced to provide "just enough" power to support different functional operations. Both dynamic and leakage power consumption can be reduced in multivoltage designs. For dynamic power, a minor adjustment to the voltage level can result in a significant reduction in power consumption, which is proportional to the square of the voltage. For leakage power, the consumption can be reduced by powering down some components when they are inactive.

Multivoltage designs involve partitioning of a chip into areas called "voltage islands" that can be operated at different voltage levels, or be turned off when idling. With the use of voltage islands, the chip design process is becoming more complicated. We need to solve the problems of island partitioning, voltage assignment, and floorplanning simultaneously under area, power, timing, and other physical constraints. These problems must be solved at the same time since their results will mutually affect each other. Also, there are other issues to be considered. For example, voltage islands should be placed close to their corresponding power pins in order to make power routing easier and to reduce $I R$ drop. Besides, each island requires level shifters to communicate with others and overhead in area and delay will be resulted. These additional issues have created many new challenges in generating floorplans for multivoltage designs. An example is shown in Fig. 1. In this example, the possible voltage levels of each core and groupings of similar inactive periods (to generate islands with power down mode) are shown on the right hand side. Assuming that the number of islands is three, one possible partitioning is to group cores $A, B$, and $C$ as one island operating at voltage $1.0-\mathrm{V}$, core $D$ on its own as one island at voltage $1.5-\mathrm{V}$ and cores $I, K, L$, and $M$ as one island at $1.2-\mathrm{V}$. Notice that other cores will be operated at the chip-level voltage. The island containing $I, K, L$, and $M$ can be powered down during sleep, as well as the island containing the single core $D$. A candidate floorplan solution for such a partitioning and voltage assignment is shown on the left.

There are several previous papers addressing this voltage island-driven floorplanning problem. One recent work is by Lee et al. [5]. Given a netlist without reconvergent fanouts, a voltage assignment (with two voltage levels of VDDL and VDDH) is first performed on the netlist according to the timing requirement before the floorplanning step. Level shifters are then inserted into the nets according to the voltage assignment 
result when a VDDL block drives a VDDH block. At the end, a power-network aware floorplanner is invoked to pack the blocks such that the power-network resource, estimated as the sum of the perimeters of the voltage islands, will be minimized. As a result, blocks in the same voltage island will be placed close to each other. In their approach, the voltage assignment step and the floorplanning step are done separately. $\mathrm{Hu}$ et al. [3] have also considered this simultaneous island partitioning, voltage assignment, and floorplanning problem in SoC designs. Simulated annealing is used as the basic searching engine. Given a candidate solution, perturbations are performed to split an island, change the voltage of an island or change all the islands of one voltage to another voltage. Chiplevel floorplanning is then performed to find a floorplan in which compatible islands (islands with the same voltage) are likely to be adjacent. An island merging process is then applied to reduce the number of islands. At the end, island-level floorplanning is done to each newly formed island to shrink its area. The whole process is repeated until a satisfactory solution is obtained. Their approach does not consider islands with power down mode and the search space is large. Mak and Chen [7] have also addressed this problem on SoC designs. Given a floorplanning input, the voltage assignment and island partitioning problem is formulated as a $0-1$ integer linear program. In their approach, a few candidate floorplan solutions are generated based on metrics like area and interconnect cost, then voltage assignment and partitioning are performed on these candidate floorplans using the integer linear programming approach to identify the best candidate solution. A fragmentation cost (number of adjacent cores operating at different voltages) is used to model the power network complexity but this cost is not related to the number of islands directly. There are some other previous works which address issues like reliability [12] and temperature reduction [4] in SoC voltage island partitioning and floorplanning. For island partitioning, Wu et al. [11] minimized the number of voltage islands after placement. Guo et al. [2] addressed the voltage assignment and voltage island generation problem in placement to minimize the number of level shifters. Cai et al. [1] proposed a voltage island generation flow in standard cell-based designs to reduce power consumption under performance constraint and to reduce layout overheads caused by cell clustering to form islands.

In this paper, we present a floorplanning method for SoC designs that is tightly integrated with island partitioning and voltage assignment. A preliminary version of it has appeared in [6]. Simulated annealing is used with normalized Polish expression (NPE) [10] as the floorplan representation. ${ }^{1}$ Our cost function now considers area, wire length, power, and level shifter usage. ${ }^{2}$ NPE is used because the slicing tree structure is a suitable data structure on which island partitioning and voltage assignment can be done optimally and efficiently. In each step of the annealing process, a candidate floorplan solution is generated on which optimal island partitioning

\footnotetext{
${ }^{1}$ Since many input cores have flexibilities in shape at this stage, the restricted use of slicing floorplan can also give satisfactory results.

${ }^{2}$ The cost function can be easily extended to consider routability by including a term on congestion estimation.
}

and voltage assignment will be performed simultaneously to compute the smallest possible power consumption for that candidate floorplan solution. This is done by dynamic programming with an efficient cost table updating technique. In this way, we can integrate the three steps closely, and reduce the searching space (instead of doing voltage assignment by the "move" operations of the annealing process as in [3]). In this floorplanning framework, we can also generate islands with power down mode to optimize the total power consumption further. We compared our approach with the most updated previous work [7] on the same problem, and results show that our approach is much more efficient and is able to save more power in most cases, while with less area overhead. Similar to [7], timing is considered according to the assumption that the given voltage levels of each core are all feasible for achieving timing closure. To further study this problem, we have developed two other methods to solve this voltage islanddriven floorplanning problem. One of them is a simplified version of the dynamic programming approach, and the other one assumes a lowest possible power consumption for each core. We compare these two methods with our approach and experimental results show that our approach can perform the best. The simplified dynamic programming approach performs a little bit inferior to our approach in terms of solution quality, with a $5.37 \%$ reduction in running time on average. The lowest possible power approach consumes less execution time and achieves maximum power saving, but the number of voltage islands generated is relatively large, while our approach can generate a solution of which the power consumption is fairly close to the lowest possible value, while the number of voltage islands is much smaller. In addition, we have also extended our floorplanner to consider the area and power overhead of level shifters, to solve a minVdd version of this voltage island driven floorplanning problem (in which each core, instead of having a set of discrete feasible voltage levels, has a minimum voltage level above which the core can be operated properly and achieve timing closure) and to consider the ease of power network routing (proximity to power pins and shapes of voltage islands). By using our approach, we can achieve significant power savings (up to 50\%) for all datasets, without any significant increase in area and wire length.

We will define the problem in Section II, we will then discuss the methodology used in Section III. The experimental results will be reported in Section VI before the conclusion and discussion in the last section.

\section{PRoblem Formulation}

In this problem, we are given a set of $n$ cores with areas $A_{1}, A_{2}, \ldots, A_{n}$ and aspect ratio bounds $\left[l_{i}, u_{i}\right]$ for $i=1, \ldots, n$. Each core $i$ is associated with a power table $T_{i}$ that specifies the legal voltage levels for the core and the corresponding average power consumption values such that the cycle time can be achieved. These tables can be obtained by simulations that try applying different supply voltages to the core. The power consumption corresponding 

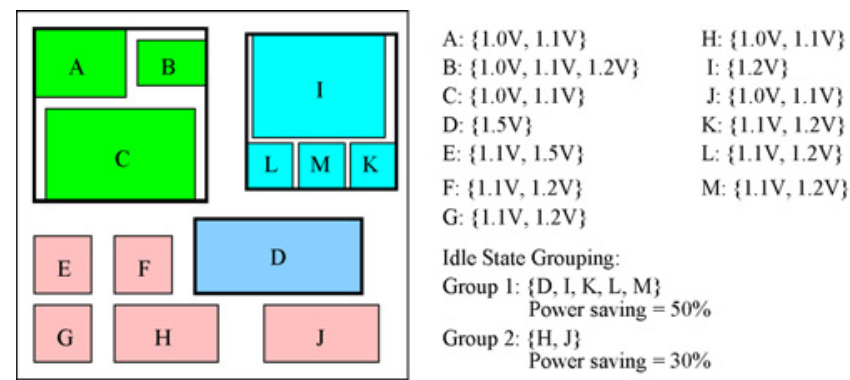

Fig. 1. Example of the voltage island driven floorplanning problem.

to each legal voltage can then be estimated. ${ }^{3}$ We are also given a set of $m$ nets $\left\{N_{1}, N_{2}, \ldots, N_{m}\right\}$ and a set of groupings $\left\{G_{1}, G_{2}, \ldots, G_{p}\right\}$ between the cores such that the cores in each group $G_{i}$ have similar inactive periods and will have a $s_{i} \%$ saving in power consumption if they are grouped together as an island with power down mode. ${ }^{4}$

Given a constant $K$ and a chip-level voltage $V_{c}$, our goal is to generate a floorplan $F$ with $K$ rectangular voltage islands so that the total power consumption is minimized. ${ }^{5}$ Each island will be supplied with the lowest possible voltage level common to all the cores in that island while the remaining cores not assigned to any island will be operated at the chip-level voltage. Islands containing blocks all belonging to the same group $G_{i}$ can have a further reduction in power consumption by $s_{i} \%$ by shutting it down during sleep. ${ }^{6}$

An alternative scenario of this problem is that each core $i$, instead of having a few discrete legal voltage levels, has a minimum voltage level $\min V_{i}$ such that $i$ can operate properly and achieve timing closure at a voltage level not less than $\min V_{i}$. We call this the $\min V d d$ version of this voltage islanddriven floorplanning problem, and will extend our discussion on this in a later part of this paper.

\section{A. Groupings of Cores With Similar Inactive Periods}

The grouping information can be generated according to the dynamic behaviors of the cores obtained from a power state machine (PSM) [8]. A PSM represents all the states a chip can operate in and the possible transitions between them. Each state in a PSM describes the operating mode of the cores. For example, a core can be active, idle or sleep in any particular state. Each state also has a weight that represents the relative amount of time the system will remain in that particular state. The larger the weight, the higher is the chance that the system

\footnotetext{
${ }^{3}$ In this paper, we assume that the power cost of a core $i$ operated at voltage $v$ is $v^{2} A_{i}$, i.e., we fill in the table according to this formulation. However, the use of a power cost table gives us flexibility in specifying the power costs of a core at different voltage levels.

${ }^{4}$ Note that this percentage of power saving is computed according to the amount of time each core can be shut down in comparison with the total computational time, and the cores with similar inactive periods are grouped together. Therefore, even if only some cores in a group form an island, there is still $s_{i} \%$ saving in power.

${ }^{5} \mathrm{We}$ assume that the given voltage levels of each core can meet timing, so we do not consider timing explicitly in our formulation.

${ }^{6} \mathrm{This}$ factor $s_{i} \%$ is the percentage saving compared with the case when the cores are not shut down at all. Therefore, this is an estimation of the amount of time (compared with the total operational time) that the computations of the cores are not needed.
}

will be in that state within a certain time window. The power to an island with power down mode must remain active if any of the cores in it is active. The relative amount of time a group of cores on the same island will remain inactive can be estimated from the PSM, and the percentage saving in power can then be estimated from it. We can exhaust different ways of groupings in a preprocessing step to find out which grouping is possible, given an estimate of the overhead cost in energy consumption and area of the power gating circuitry. Those potentially beneficial groups will form the $G_{i}$ s in the above formulation.

\section{Methodology}

Our floorplanner is based on simulated annealing using NPE as the representation. For each candidate floorplan solution represented by an NPE, we will perform an optimal island partitioning and voltage assignment to maximize the total power saving. The cost function of the annealing process is to minimize a weighted sum of the area, wire length, and power. We can also extend our floorplanner to consider level shifters and proximity to power pins. Details will be given in the following sections.

\section{A. Optimal Island Partitioning and Voltage Assignment}

Given a candidate floorplan solution represented by a NPE, we can construct the corresponding slicing tree and perform optimal island partitioning and voltage assignment on it. This can be done efficiently by dynamic programming. The pseudocode is shown below.

Pseudocode TreePart( $u, k)$

// Partition the subtree under node $u$ into $k$ subtrees to // minimize the total power consumption such that the cores // (leaf nodes) in each of these $k$ subtree will form one island

// operated at one common voltage possibly with power down

// mode while the remaining cores not belonging to any of // these $k$ subtrees will be operated at chip-level voltage $V_{c}$

1. min cost $=\infty$

2. If $k \overline{i s} 0$, return( $\operatorname{power}(u))$.

3. If cost_table $[u][k]$ is updated, return(cost_table $[u][k])$.

4. If $k$ is $\bar{l}$,

5. $C_{1}=$ TreePart $(\operatorname{lchild}(u), 1)+\operatorname{power}(\operatorname{rchild}(u))$

6. $\quad C_{2}=$ TreePart $(\operatorname{rchild}(u), 1)+\operatorname{power}(\operatorname{lchild}(u))$

7. $C_{3}=$ non Subtree $(u, 1)$

8. $C_{4}=\operatorname{cost}(\{u\})$

9. $\min$ cost $=\min \left\{C_{1}, C_{2}, C_{3}, C_{4}\right\}$

10. Store min_cost into cost_table $[u][1]$.

11. Return (min cost).

12. Else

13. min cost $=$ non Subtree $(u, k)$

14. For $\bar{i}=0$ to $k$

15. $\quad C=$ TreePart $(\operatorname{lchild}(u), i)+$ Tree Part $($ rchild $(u), k-i)$

16. If $\min$ cost $>C$, $\min$ cost $=C$

17. Store min _cost into cost_table $[u][k]$.

18. Return(min cost). 
At the beginning, Tree Part (root, $k$ ) is called to obtain an optimal island partitioning and voltage assignment of the whole floorplan, where root is the root of the slicing tree corresponding to the NPE under consideration and $k$ is the number of voltage islands we want to produce. When $k$ is zero (line 2), no voltage island is formed in the subtree of $u$, so the power consumption $\operatorname{power}(u)$ will be computed as $\left(V_{c}\right)^{2} A_{\{u\}}$, where $V_{c}$ is the chip-level voltage and $A_{\{u\}}$ is the total area of the cores in the subtree of $u$. For nonzero $k$, we will first check whether this optimal cost has been computed before and is available in the table for immediate return (line 3). If this value is not available, we will consider the situations when $k$ is one and when $k$ is larger than one separately. When $k$ is one (line 4), there are four cases.

1) Case 1 (line 5), we continue to search for a voltage island in the left subtree of $u$ and let the right subtree operates at the chip-level voltage $V_{c}$.

2) Case 2 (line 6), similarly, we look for a voltage island in the right subtree of $u$ and let all the cores in the left subtree work at $V_{c}$.

3) Case 3 (line 7), use the function nonSubtree () to group the cores across a number of subtrees along the left tree branches of $u$ into a voltage island (details will be given in the next section).

4) Case 4 (line 8), the entire subtree $u$ is regarded as one voltage island and the power consumption $\operatorname{cost}(\{u\})$ will be computed as

$$
\operatorname{cost}(\{u\})=\left(v_{\{u\}}\right)^{2} A_{\{u\}}
$$

where $v_{\{u\}}$ is the smallest common voltage among all the cores in the subtree rooted at $u$ and $A_{\{u\}}$ is the total area of the cores in the subtree rooted at $u$.

We will compute the costs of the four cases, respectively, and the smallest one will be returned and recorded in the table for future use. When $k$ is more than one (line 12), we will recursively call the procedure TreePart() to exhaust all the possible partitionings of the subtree of $u$, including both intersubtree partitionings (line 13) and intra-subtree ones (lines 1416). The minimum one will be returned and recorded in the table.

1) Voltage Islands in nonSubtrees: Notice that a voltage island (a rectangular region) may be formed by a set of contiguous right subtrees linked by internal nodes of the same type. An example is shown in Fig. 2. Therefore, we need the procedure nonSubtree() to enumerate these cases. The pseudocode of nonSubtree() is shown in the following. In this procedure, we exhaust all the cases of forming one island with two or more contiguous right subtrees and the one with the smallest power consumption will be returned. On line 7, we compute the cost of grouping the right subtrees in $S$ as one island and having the remaining $k-1$ islands in the last left subtree (subtree $D$ in the example of Fig. 2).

Pseudocode nonSubtree $(u, k)$

// Exhaust the cases of forming one island by a number // of contiguous right subtrees, while the remaining $k-1$ // islands are formed in the remaining left subtree.

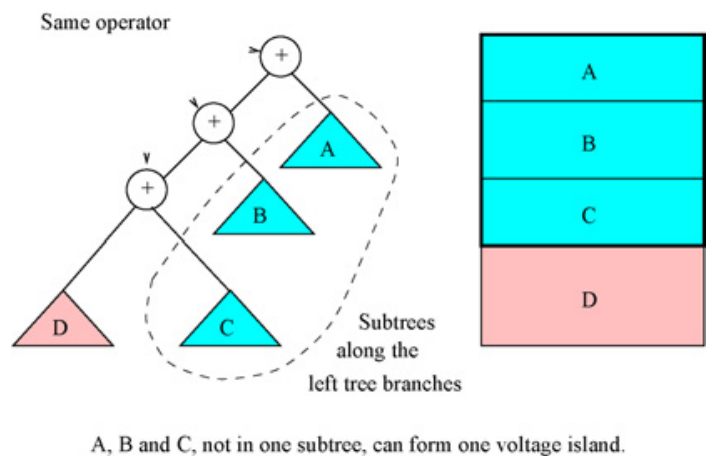

Fig. 2. Example of forming island across subtrees.

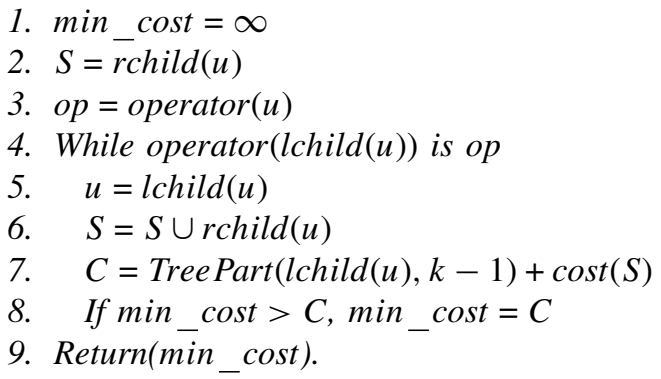

2) Proof of Optimality: The procedure TreePart() will give the optimal partitioning to minimize the total power consumption. Given a candidate floorplan solution represented by a normalized slicing tree rooted at $u$ and the number of voltage islands required $k$, TreePart() will exhaust all the possible cases recursively and return the best solution. When $k$ is zero, there is only one case that all the cores in the tree rooted at $u$ (called $T_{u}$ ) are operated at the chip voltage. When $k$ is one, there is only one voltage island among all the cores in $T_{u}$. The first three cases are obvious: 1 ) the island is in the left subtree of $u ; 2$ ) the island is in the right subtree; and 3) all the cores in $T_{u}$ form one island. There is still a case that the island is formed between the left and right subtrees of $u$. This may happen only when two consecutive internal nodes are of the same type (both “+” or both “*”). In a normalized slicing tree, an internal node will not be of the same type as its right child, so this will happen only along the left branch. nonSubtree() will exhaust this last case of forming one island by a set of contiguous right subtrees along the left branch rooted at $u$. When $k$ is larger than one, the cases are similar to those when $k$ is one and TreePart() will exhaust all different ways of distributing the $k$ islands between the left and right subtrees of $u$ and the case of having an island lying between the two subtrees. Since TreePart() has exhausted all different cases of forming $k$ islands in a given candidate floorplan, the solution returned by Tree Part () is optimal.

3) Handling Island With Power Down Mode: Islands with power down mode can be easily handled in our framework. When computing the power consumption of an island formed with the cores in the set of subtrees rooted at a node in set $X$ by calling $\operatorname{cost}(X)$ in the procedure TreePart() and non Subtree(), we only need to check if all the cores belong to one group $G_{i}$ 
for some $i=1, \ldots, p$. If this is true, the island formed can be shut down during sleep and have an additional power saving of $s_{i} \% .^{7}$ Notice that an island containing just one single core can also be shut down during sleep mode if the core belongs to some idle state group. In this way, our floorplanner can give optimal island partitioning and voltage assignment taking into account islands with power down mode given any candidate floorplan solution.

4) Speedup in Implementation and Complexity: A table cost_table $[v][j]$ for $j=1, \ldots, K$ is kept at each internal node $v$ of the slicing tree to record the optimal power consumption of partitioning the cores in the subtree rooted at $v$ into $j$ islands. This data structure can help to minimize the number of recursive calls and to avoid repetitive computations. It can be seen from the procedure TreePart ( ) that whenever we want to find the optimal power saving at a node $u$ with $k$ voltage islands, we will first check whether this is computed before and the required information is available from cost table $[u][k]$. If it is available, the optimal value is returned immediately (line 3). Otherwise, it is computed recursively and the computed value will be saved in the cost_table to be used in some later steps (lines 10 and 17). After a move in the annealing process, only a small part of the whole slicing tree will be changed and we only need to update the tables of the affected nodes once. The affected nodes will be those lying on the paths from the modified parts of the tree to the root. For those affected nodes, the corresponding cost_tables will be flagged as "not updated" and will be updated during the recursive calls.

The size of the cost table is $O(n K)$ where $n$ is the number of cores and $K$ is the number of voltage islands, because each internal node $v$ has $K$ entries in the table, cost_table $[v][j]$ for $j=1, \ldots, K$, and there are $n$ internal nodes in total. For each affected node $v$, we need to update all the $K$ entries of its table once. Since each entry is just updated once, the time complexity will be the same as that of updating all the affected nodes in a bottom-up fashion from the leaves to the root. If the nodes were updated from the leaves to the root, the time taken to update a table at a node $v$ was $O\left(K^{2}\right)$, because there were $K$ entries and each entry took $O(K)$ time (the tables of $v$ 's children have already been updated). Therefore the total time to perform all the updates in each iteration is (number of affected nodes ) $\times O\left(K^{2}\right)$. This is $O\left(K^{2} n\right)$ in the worst case, and is $\left(K^{2} \log n\right)$ on average.

\section{B. Annealing Schedule}

In our annealing engine, the temperature is set to $10^{5}$ at the beginning and will drop at a rate of 0.95 . At each temperature, $30 * n$ random moves are performed, where $n$ is the number of blocks in the dataset. The annealing process stops either when less than 0.5 percent random moves are accepted at a certain temperature, or when the temperature falls below $10^{-5}$.

\footnotetext{
${ }^{7}$ Even if some cores in the group are not in the island, we assume that there is still $s_{i} \%$ saving in power since this percentage saving is computed based on the amount of time each core can be shut down in comparison with the total computational time. However, the way to calculate this saving in power can be modified for different problem formulations.
}

\section{Moves}

There are three kinds of moves to change the NPE of a candidate floorplan solution during annealing. This set of moves has been proven to be complete to change any arbitrary solution to any other arbitrary solution.

1) Swap: Swap two adjacent blocks.

2) Complement: Complement a chain of operators.

3) SwapOp: Swap a block with its adjacent operator.

\section{Cost Function}

We use the cost function $\psi=A+\lambda_{w} W+\lambda_{p} P$ to evaluate a floorplan where $A$ is the area of the floorplan, $W$ is the total wire length estimated by the half perimeter bounding box and $P$ is the total power consumption. The parameters $\lambda_{w}$ and $\lambda_{p}$ are the weights which will be set at the beginning of the annealing process by random walks to make the three terms similar in weighting. Note that this cost function can be modified to consider routability (by having an additional term on congestion estimation) or the fixed-outline constraint (by replacing the area term $A$ by $\lambda_{\infty}\left(\max \left\{0, w-W^{\prime}\right\}+\max \{0, h-\right.$ $\left.H^{\prime}\right\}$ ) where $\lambda_{\infty}$ is a large positive constant, $w$ and $h$ are the width and height of the candidate floorplan solution, and $W^{\prime}$ and $H^{\prime}$ are the fixed width and height required for the final floorplan design).

\section{EXTENSION to BASIC Methodology}

Based on the framework discussed in the above Section III, the methodology can be modified to handle the following varied versions of the problem, minVdd optimization and adjustable background voltage.

\section{A. MinVdd Optimization}

As discussed in the problem formulation section, an alternative scenario of this problem is that each core $i$, instead of having a few discrete legal voltage levels, has a minimum voltage $\min V_{i}$ such that $i$ can operate properly and achieve timing closure at a voltage level not less than $\min V_{i}$. Our general framework can be readily modified to address this minVdd version of the problem. The major modification made to the dynamic programming is that when we get down to the base case that the whole subtree forms only one island, instead of finding the lowest voltage level legal to all cores in the island, we should compute the highest $\min V_{i}$ among all the cores $i$ in the island and take that as the voltage level being used in that island. We can achieve this by computing the function $\operatorname{cost}(\{u\})$ of a subtree $u$ as follows:

$$
\operatorname{cost}(\{u\})=A_{\{u\}} \times \max _{\text {core } i \in u} \min V_{i}^{2} .
$$

Notice that since we can control the number of voltage islands by adjusting the parameter $k$ in the procedure Tree Part $($ root,$k)$, the floorplanning process will not end up with getting more voltage levels than desired. That means, the algorithm has already taken into account the maximum number of voltage levels allowed to find an optimal way of partitioning and voltage assignment such that the total power consumption is minimized. 
TABLE I

DATASETS

\begin{tabular}{|l|c|c|c|}
\hline Data & $\begin{array}{c}\text { Block } \\
\text { No. }\end{array}$ & $\begin{array}{c}\text { Net } \\
\text { No. }\end{array}$ & Groups \\
\hline$n 10$ & 10 & 118 & $3(30 \%)$ \\
\hline$n 30$ & 30 & 349 & $5(30 \%), 5(20 \%)$ \\
\hline$n 50$ & 50 & 485 & $5(50 \%), 5(30 \%), 5(20 \%)$ \\
\hline$n 100$ & 100 & 885 & $10(70 \%), 5(50 \%), 6(40 \%)$ \\
\hline$n 200$ & 200 & 1585 & $10(50 \%), 10(40 \%), 9(30 \%), 9(20 \%)$ \\
\hline$n 300$ & 300 & 1893 & $15(60 \%), 15(50 \%), 15(40 \%), 15(30 \%)$ \\
\hline
\end{tabular}

\section{B. Adjustable Background Voltage}

In the problem formulation, it is assumed that a background chip-level voltage $V_{c}$ is given. Our framework can also be used to address an extended version of the problem in which the background voltage is not fixed yet. ${ }^{8}$ The final chip-level voltage is determined by the minimum (or maximum) feasible voltage level among all the cores which are not grouped into any voltage island. To achieve this, we need to expand the table at each node $L$ times where $L$ is the total number of possible voltage levels among all the cores. In this case, each entry cost_table $[v][j]\left[V_{l}\right]$ will be the optimal power consumption of partitioning the cores in the subtree rooted at $v$ into $j$ islands when the background chip-level voltage is $V_{l}$ where $1 \leq l \leq L$. Notice that some $V_{l}$ where $1 \leq l \leq L$ may be impossible to be used as the background voltage in some cases and we can use a very large cost value to denote these cases. Besides the changes made to cost_table, the procedures power(), TreePart(), and nonSubtree() will also be modified to return an array of $L$ values corresponding to the $L$ cases of using a different background voltage. Steps 910 and steps $15-17$ in the pseudocode $\operatorname{TreePart}(u, k)$ will all become array operations treating the $L$ different choices of background voltages separately and correspondingly.

All entries in the tables corresponding to different background voltages can be updated simultaneously during the recursive calls and the run time is thus only linearly scaled up by $L$. This is very affordable in practice since the number of possible voltage levels $L$ is usually small. The memory size of such expanded cost table will be $O(n K L)$.

\section{Alternatives to Solve the Problem}

To further study the problem, we have developed two other methods to solve the same voltage island-driven floorplanning problem. One of them is a simplified version of the dynamic programming approach described in Section III-A, and the other one assumes a lowest possible power consumption for each module.

\section{A. Simplified Dynamic Programming Approach}

In this simplified dynamic programming approach, the islands formed across the left and right subtrees are not taken into account, i.e., step 7 and step 13 in the pseudocode of

\footnotetext{
${ }^{8}$ This feature may be useful when this floorplanning tool is used as a prototype floorplanner during the front-end designing stages when the background voltage is not fixed yet.
}

TABLE II

COMPARISONS WITH A PREVIOUS WORK [7]

\begin{tabular}{|l|c|c|c|c|c|c|}
\hline \multirow{2}{*}{ Dataset } & \multicolumn{2}{|c|}{$\begin{array}{c}\text { Power Saving } \\
(\%)\end{array}$} & \multicolumn{2}{c|}{$\begin{array}{c}\text { Dead } \\
\text { Space }(\%)\end{array}$} & \multicolumn{2}{c|}{$\begin{array}{c}\text { Run Time* } \\
\text { (s) }\end{array}$} \\
\cline { 2 - 7 } & Ours & {$[7]$} & Ours & {$[7]$} & Ours & {$[7]$} \\
\hline apte & 51.64 & 53.78 & 2.341 & 3.422 & 1.457 & 5.482 \\
\hline xerox & 40.45 & 22.85 & 3.667 & 5.259 & 1.335 & 7.079 \\
\hline hp & 41.34 & 25.37 & 3.855 & 5.700 & 1.570 & 122.9 \\
\hline ami33 & 48.69 & 44.12 & 4.376 & 5.784 & 19.43 & 89.39 \\
\hline ami49 & 47.21 & 41.13 & 4.840 & 6.440 & 68.78 & 90.46 \\
\hline 2xerox & 43.68 & 33.53 & 5.136 & 3.765 & 10.63 & 74.75 \\
\hline 2hp & 39.58 & 17.47 & 4.880 & 5.650 & 9.55 & 26.86 \\
\hline ami75 & 47.95 & 39.06 & 6.552 & 6.330 & 166.9 & 316.5 \\
\hline ami99 & 46.33 & 41.16 & 8.630 & 7.666 & 298.5 & 684.4 \\
\hline ami200 & 42.68 & 41.90 & 9.110 & 10.88 & 1722 & 3851 \\
\hline ami300 & 41.85 & 40.69 & 10.90 & 12.02 & 3061 & 7380 \\
\hline Average & 44.67 & 36.46 & 5.844 & 6.655 & 487.4 & 1150 \\
\hline
\end{tabular}

*[7] is run on a Linux with a $2.1 \mathrm{GHz} \mathrm{CPU}$ and $4 \mathrm{~GB}$ RAM.

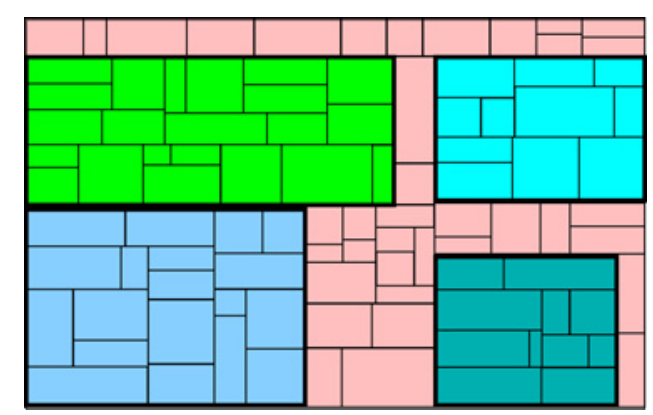

Fig. 3. One resultant floorplan of $n 100$ with four voltage islands.

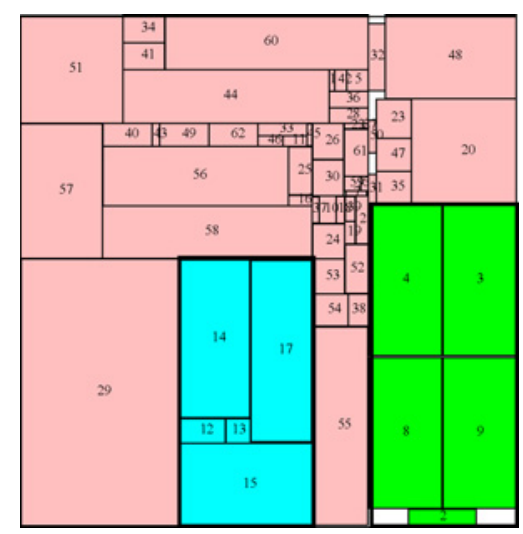

Fig. 4. One resultant floorplan of playout with some blocks operated at very low voltages.

the procedure TreePart $(u, k)$ in Section III-A are removed. This approach is clearly suboptimal for a candidate floorplan, ${ }^{9}$ since the solutions that contain islands formed across subtrees are neglected. However, the solution quality may not be significantly degraded by this simplification, since the potentially optimal grouping of some cores across subtrees in one candidate floorplan is very likely to be generated in

\footnotetext{
${ }^{9}$ This approach is suboptimal in terms of power saving with respect to a particular candidate floorplan, but the overall simulated annealing-based optimization using this simplified approach may sometimes produce a better solution due to the randomness of the annealing process.
} 
TABLE III

EXPERIMENTAL RESULTS WITH IDLE ISLANDS

\begin{tabular}{|c|c|c|c|c|c|c|c|c|c|c|c|c|c|c|c|}
\hline Data & $K$ & $\begin{array}{c}\text { Total } \\
\text { Power } \\
\left(x_{1}\right)\end{array}$ & $\begin{array}{c}\text { Power } \\
\text { Saving } \\
(\%)\end{array}$ & $\begin{array}{c}\text { Dead } \\
\text { Space } \\
(\%) \\
\left(x_{2}\right)\end{array}$ & $\begin{array}{c}\text { Wire } \\
\text { Length } \\
(\times 10) \\
\left(x_{3}\right)\end{array}$ & $\begin{array}{l}\text { No. of } \\
\text { Idle } \\
\text { Islands }\end{array}$ & $\begin{array}{l}\text { Run } \\
\text { Time } \\
(\mathrm{s}) \\
\left(x_{4}\right) \\
\end{array}$ & Data & $K$ & $\begin{array}{c}\text { Total } \\
\text { Power } \\
\left(x_{1}\right)\end{array}$ & $\begin{array}{c}\text { Power } \\
\text { Saving } \\
(\%)\end{array}$ & $\begin{array}{c}\text { Dead } \\
\text { Space } \\
(\%) \\
\left(x_{2}\right) \\
\end{array}$ & $\begin{array}{c}\text { Wire } \\
\text { Length } \\
(\times 10) \\
\left(x_{3}\right)\end{array}$ & $\begin{array}{l}\text { No. of } \\
\text { Idle } \\
\text { Islands }\end{array}$ & $\begin{array}{c}\text { Run } \\
\text { Time } \\
(\mathrm{s}) \\
\left(x_{4}\right)\end{array}$ \\
\hline \multirow[t]{7}{*}{$n 10$} & 0 & 498778 & 0.000 & 0.394 & 1185 & 0 & 3.12 & \multirow[t]{7}{*}{$n 100$} & 0 & 403877 & 0.000 & 0.638 & 13028 & 0 & 427.86 \\
\hline & 1 & 393660 & 21.075 & 0.296 & 1288 & 0 & 3.81 & & 1 & 332093 & 17.774 & 0.827 & 12997 & 0 & 409.72 \\
\hline & 2 & 334853 & 32.865 & 0.748 & 1314 & 1 & 4.32 & & 2 & 303367 & 24.886 & 0.600 & 13435 & 0 & 416.05 \\
\hline & 3 & 277862 & 44.291 & 0.748 & 1554 & 1 & 2.99 & & 3 & 256686 & 36.445 & 4.530 & 13576 & 0 & 475.06 \\
\hline & 4 & 267263 & 46.416 & 1.561 & 1350 & 1 & 5.41 & & 4 & 232262 & 42.492 & 1.599 & 14310 & 0 & 508.93 \\
\hline & 5 & 267263 & 46.416 & 0.487 & 1277 & 2 & 3.62 & & 5 & 231121 & 42.774 & 3.138 & 13882 & 1 & 572.86 \\
\hline & 6 & 267263 & 46.416 & 0.407 & 1358 & 2 & 4.36 & & 6 & 224879 & 44.320 & 3.364 & 14309 & 1 & 597.98 \\
\hline \multirow[t]{7}{*}{$n 30$} & 0 & 469330 & 0.000 & 0.732 & 3341 & 0 & 32.73 & \multirow[t]{7}{*}{$n 200$} & 0 & 395316 & 0.000 & 0.808 & 29421 & 0 & 1600.22 \\
\hline & 1 & 312071 & 33.507 & 0.848 & 3477 & 0 & 42.30 & & 1 & 341132 & 13.706 & 6.063 & 31139 & 0 & 1762.17 \\
\hline & 2 & 221819 & 52.737 & 0.729 & 3629 & 1 & 37.43 & & 2 & 309471 & 21.716 & 1.490 & 30480 & 0 & 1770.16 \\
\hline & 3 & 242060 & 48.424 & 0.760 & 3836 & 2 & 46.22 & & 3 & 259555 & 34.342 & 3.154 & 30820 & 0 & 1814.28 \\
\hline & 4 & 221819 & 52.737 & 4.136 & 3803 & 2 & 43.69 & & 4 & 246987 & 37.522 & 5.704 & 32004 & 0 & 2080.43 \\
\hline & 5 & 221819 & 52.737 & 4.107 & 4006 & 2 & 59.83 & & 5 & 261257 & 33.912 & 3.426 & 30720 & 1 & 2036.82 \\
\hline & 6 & 216165 & 53.942 & 0.701 & 3823 & 2 & 57.28 & & 6 & 247342 & 37.432 & 0.892 & 32668 & 1 & 2058.11 \\
\hline \multirow[t]{7}{*}{$n 50$} & 0 & 446803 & 0.000 & 0.744 & 8001 & 0 & 97.72 & \multirow[t]{7}{*}{$n 300$} & 0 & 614632 & 0.000 & 2.527 & 49839 & 0 & 3295.63 \\
\hline & 1 & 267143 & 40.210 & 3.629 & 8347 & 0 & 110.85 & & 1 & 541437 & 11.910 & 1.975 & 49289 & 0 & 3499.20 \\
\hline & 2 & 246592 & 44.810 & 6.623 & 8348 & 0 & 138.71 & & 2 & 494289 & 19.580 & 2.166 & 50565 & 0 & 3398.88 \\
\hline & 3 & 224870 & 49.671 & 2.141 & 8888 & 1 & 128.57 & & 3 & 436625 & 28.960 & 1.961 & 51298 & 0 & 3576.74 \\
\hline & 4 & 202117 & 54.764 & 1.750 & 8296 & 2 & 140.25 & & 4 & 457999 & 25.484 & 3.514 & 50383 & 0 & 3931.90 \\
\hline & 5 & 227838 & 49.007 & 0.775 & 8604 & 1 & 138.53 & & 5 & 379049 & 38.329 & 1.151 & 50636 & 0 & 3843.81 \\
\hline & 6 & 200281 & 55.175 & 0.878 & 8299 & 3 & 135.08 & & 6 & 386475 & 37.120 & 2.677 & 52048 & 1 & 4077.38 \\
\hline
\end{tabular}

TABLE IV

EXPERIMENTAL RESUlTS FOR SIMPLIFIED DYNAMIC PROGRAMMING APPROACH

\begin{tabular}{|c|c|c|c|c|c|c|c|c|c|c|c|c|c|c|c|}
\hline Data & $K$ & $\begin{array}{c}\text { Total } \\
\text { Power } \\
\left(y_{1}\right)\end{array}$ & $\begin{array}{c}\text { Power } \\
\text { Saving } \\
(\%)\end{array}$ & $\begin{array}{c}\text { Dead } \\
\text { Space } \\
(\%) \\
\left(y_{2}\right)\end{array}$ & $\begin{array}{c}\text { Wire } \\
\text { Length } \\
(\times 10) \\
\left(y_{3}\right)\end{array}$ & $\begin{array}{l}\text { No. of } \\
\text { Idle } \\
\text { Islands }\end{array}$ & $\begin{array}{l}\text { Run } \\
\text { Time } \\
(s) \\
\left(y_{4}\right)\end{array}$ & Data & $K$ & $\begin{array}{c}\text { Total } \\
\text { Power } \\
\left(y_{1}\right)\end{array}$ & $\begin{array}{c}\text { Power } \\
\text { Saving } \\
(\%)\end{array}$ & $\begin{array}{c}\text { Dead } \\
\text { Space } \\
(\%) \\
\left(y_{2}\right)\end{array}$ & $\begin{array}{c}\text { Wire } \\
\text { Length } \\
(\times 10) \\
\left(y_{3}\right)\end{array}$ & $\begin{array}{c}\text { No. of } \\
\text { Idle } \\
\text { Islands }\end{array}$ & $\begin{array}{c}\text { Run } \\
\text { Time } \\
(\mathrm{s}) \\
\left(y_{4}\right)\end{array}$ \\
\hline \multirow[t]{7}{*}{$n 10$} & 0 & 498778 & 0.000 & 0.394 & 1185 & 0 & 3.08 & \multirow[t]{7}{*}{$n 100$} & 0 & 403877 & 0.000 & 0.638 & 13028 & 0 & 424.38 \\
\hline & 1 & 393660 & 21.075 & 0.345 & 1337 & 0 & 3.36 & & 1 & 293126 & 27.422 & 1.289 & 12973 & 0 & 454.97 \\
\hline & 2 & 334853 & 32.865 & 5.801 & 1422 & 1 & 3.12 & & 2 & 274892 & 31.937 & 1.081 & 13855 & 0 & 413.25 \\
\hline & 3 & 297459 & 40.362 & 1.320 & 1573 & 1 & 3.89 & & 3 & 283353 & 29.842 & 3.358 & 13827 & 0 & 511.74 \\
\hline & 4 & 267263 & 46.416 & 0.759 & 1467 & 1 & 4.26 & & 4 & 244486 & 39.465 & 0.695 & 14161 & 0 & 433.60 \\
\hline & 5 & 267263 & 46.416 & 0.875 & 1451 & 1 & 4.90 & & 5 & 239411 & 40.722 & 4.899 & 14305 & 1 & 510.97 \\
\hline & 6 & 267263 & 46.416 & 1.566 & 1301 & 1 & 4.29 & & 6 & 225095 & 44.266 & 2.687 & 14735 & 1 & 474.18 \\
\hline \multirow[t]{7}{*}{$n 30$} & 0 & 469330 & 0.000 & 0.732 & 3341 & 0 & 32.47 & \multirow[t]{7}{*}{$n 200$} & 0 & 395316 & 0.000 & 0.808 & 29421 & 0 & 1588.19 \\
\hline & 1 & 312071 & 33.507 & 0.993 & 3543 & 0 & 33.94 & & 1 & 325408 & 17.684 & 6.124 & 33170 & 0 & 1707.47 \\
\hline & 2 & 231225 & 50.733 & 2.224 & 3690 & 1 & 39.28 & & 2 & 324624 & 17.883 & 3.271 & 31690 & 0 & 1673.27 \\
\hline & 3 & 243983 & 48.015 & 1.159 & 3905 & 1 & 35.10 & & 3 & 306767 & 22.399 & 7.035 & 31673 & 0 & 1667.41 \\
\hline & 4 & 215589 & 54.065 & 6.933 & 3892 & 1 & 42.56 & & 4 & 251292 & 36.433 & 3.809 & 33361 & 0 & 1727.21 \\
\hline & 5 & 209498 & 55.362 & 0.815 & 3702 & 2 & 44.39 & & 5 & 264266 & 33.151 & 3.292 & 32422 & 1 & 1957.23 \\
\hline & 6 & 213404 & 54.530 & 2.909 & 3995 & 3 & 51.93 & & 6 & 261002 & 33.976 & 5.786 & 34124 & 1 & 2036.72 \\
\hline \multirow[t]{7}{*}{$n 50$} & 0 & 446803 & 0.000 & 0.744 & 8001 & 0 & 96.94 & \multirow[t]{7}{*}{$n 300$} & 0 & 614632 & 0.000 & 2.527 & 49839 & 0 & 3298.97 \\
\hline & 1 & 265290 & 40.625 & 0.940 & 8402 & 0 & 102.77 & & 1 & 538237 & 12.429 & 5.138 & 51053 & 0 & 3473.72 \\
\hline & 2 & 248471 & 44.389 & 2.010 & 8390 & 0 & 112.04 & & 2 & 487268 & 20.722 & 1.134 & 49992 & 0 & 3270.86 \\
\hline & 3 & 247515 & 44.603 & 2.544 & 8593 & 0 & 111.37 & & 3 & 454473 & 26.057 & 2.877 & 51498 & 0 & 3373.43 \\
\hline & 4 & 214415 & 52.011 & 1.679 & 8024 & 2 & 130.33 & & 4 & 435452 & 29.152 & 2.246 & 51824 & 0 & 3154.60 \\
\hline & 5 & 206536 & 53.775 & 3.166 & 8660 & 2 & 123.92 & & 5 & 395715 & 35.620 & 2.449 & 51507 & 0 & 3951.88 \\
\hline & 6 & 197507 & 55.795 & 7.718 & 8702 & 3 & 141.29 & & 6 & 407159 & 33.750 & 4.654 & 53000 & 1 & 4042.81 \\
\hline
\end{tabular}

another one in which these cores are lying in the same subtree, due to the stochastic property of simulated annealing.

\section{B. Lowest Possible Power Approach}

In this lowest possible power approach, each core is set to operate at its lowest legal working voltage level, so that the largest possible power saving (without power down mode) can be achieved. The dynamic programming procedure in this approach then simply need to count the number of voltage islands required for each candidate floorplan, and this number will replace the power consumption term in the cost function of the annealing process. This counting can be done recursively and the pseudocode is shown below. The variables Llevel and Rlevel are used for possibly merging of two neighboring islands of the same voltage level, which are separated into two subtrees of one parent node in the slicing tree. We can easily see that the run time complexity is $O(n)$ where $n$ is the number of cores, and this complexity is the smallest possible.

Pseudocode IslandCount ( $u$, Llevel, Rlevel)

// Assume that each core $i$ is already assigned a voltage // level $v_{i}$. This procedure counts the number of voltage 
// islands in the subtree rooted at $u$. Llevel and Rlevel

// are variables to be set and passed out of the procedure.

1. If $u$ contains a single core $i$

2. Llevel $=$ Rlevel $=v_{i}$

3. $\operatorname{return}(1)$

4. Else

5. $o p=$ operator $(u)$

6. $\quad a=\operatorname{IslandCount}\left(\operatorname{lchild}(u), L_{1}, R_{1}\right)$

7. $\quad b=$ IslandCount $\left(\operatorname{rchild}(u), L_{2}, R_{2}\right)$

8. If $a$ is 1

9. $x=R_{1} / /$ In this case, $L_{1}=R_{1}$

10. Llevel $=L_{1}$

11. Else

12. If operator (lchild $(u)$ ) equals op

13. $x=R_{1}$

14. Llevel $=L_{1}$

15. Else

16. $x=0$

17. Llevel $=0$

18. If $b$ is 1

19. $y=L_{2} / /$ In this case, $L_{2}=R_{2}$

20. Rlevel $=R_{2}$

21. Else

22. If operator (rchild $(u))$ equals op

23. $y=L_{2}$

24. Rlevel $=R_{2}$

25. Else

26. $y=0$

27. $\quad$ Rlevel $=0$

28. If $x \neq 0$ and $y \neq 0$ and $x$ equals $y$

29. $\operatorname{return}(a+b-1)$

30. Else

31. $\operatorname{return}(a+b)$

\section{EXPERIMENTAL RESULTS}

We have done experiments on the GSRC floorplanning benchmarks. Since no voltage information is provided in those benchmarks, we have randomly generated the voltage levels for each block from the set $\{1.0-\mathrm{V}, 1.1-\mathrm{V}, 1.2-\mathrm{V}, 1.3-\mathrm{V}$, $1.5-\mathrm{V}\}$ and $1.5-\mathrm{V}$ is assumed to be the chip-level voltage. In each dataset, groups of blocks with similar inactive periods are also randomly generated. Table I shows the details of each dataset, the fourth column indicates the grouping information of similar inactive periods, e.g., for $n 30$, there are two groups: one contains five cores with $30 \%$ power saving if being grouped together, and the other one contains five cores with $20 \%$ power saving if being grouped together. This grouping information is now generated randomly. ${ }^{10}$ Our algorithm is implemented in $\mathrm{C}$ language and all the experiments are performed on a Sun Blade 2500 with a $1.6 \mathrm{GHz} \mathrm{CPU}$ and $2 \mathrm{~GB}$ RAM.

\footnotetext{
${ }^{10}$ This grouping information can be calculated from a task graph that shows when and how long certain cores can be powered down.
}

TABLE V

COMPARISON BETWEEN OUR APPROACH AND THE SIMPLIFIED DYNAMIC PROGRAMMING APPROACH

\begin{tabular}{|c|c|c|c|c|c|}
\hline Data & $K$ & $\begin{array}{l}\text { Change in } \\
\text { Total Power } \\
(\%) \\
\left(\frac{y_{1}-x_{1}}{x_{1}}\right)\end{array}$ & $\begin{array}{c}\text { Change in } \\
\text { Total Area } \\
(\%) \\
\left(\frac{y_{2}-x_{2}}{x_{2}}\right)\end{array}$ & $\begin{array}{l}\text { Change in } \\
\text { Wire Length } \\
(\%) \\
\left(\frac{y_{3}-x_{3}}{x_{3}}\right)\end{array}$ & $\begin{array}{c}\text { Change in } \\
\text { Run Time } \\
(\%) \\
\left(\frac{y_{4}-x_{4}}{x_{4}}\right)\end{array}$ \\
\hline$n 10$ & $\begin{array}{l}0 \\
1 \\
2 \\
3 \\
4 \\
5 \\
6\end{array}$ & $\begin{array}{l}0.00 \\
0.00 \\
0.00 \\
7.05 \\
0.00 \\
0.00 \\
0.00\end{array}$ & $\begin{array}{c}0.00 \\
0.05 \\
5.36 \\
0.58 \\
-0.81 \\
0.39 \\
1.18\end{array}$ & $\begin{array}{c}0.00 \\
3.80 \\
8.22 \\
1.22 \\
8.67 \\
13.63 \\
-4.20\end{array}$ & $\begin{array}{c}-1.28 \\
-11.81 \\
-27.78 \\
30.10 \\
-21.26 \\
35.36 \\
-1.61\end{array}$ \\
\hline$n 30$ & $\begin{array}{l}0 \\
1 \\
2 \\
3 \\
4 \\
5 \\
6\end{array}$ & $\begin{array}{c}0.00 \\
0.00 \\
4.24 \\
0.79 \\
-2.81 \\
-5.55 \\
-1.28 \\
\end{array}$ & $\begin{array}{c}0.00 \\
0.15 \\
1.53 \\
0.40 \\
3.01 \\
-3.32 \\
2.27 \\
\end{array}$ & $\begin{array}{c}0.00 \\
1.90 \\
1.68 \\
1.80 \\
2.34 \\
-7.59 \\
4.50 \\
\end{array}$ & $\begin{array}{c}-0.79 \\
-19.76 \\
4.94 \\
-24.06 \\
-2.59 \\
-25.81 \\
-9.34\end{array}$ \\
\hline$n 50$ & $\begin{array}{l}0 \\
1 \\
2 \\
3 \\
4 \\
5 \\
6\end{array}$ & $\begin{array}{c}0.00 \\
-0.69 \\
0.76 \\
10.07 \\
6.08 \\
-9.35 \\
-1.39\end{array}$ & $\begin{array}{c}0.00 \\
-2.72 \\
-4.71 \\
0.41 \\
-0.07 \\
2.47 \\
7.41\end{array}$ & $\begin{array}{c}0.00 \\
0.66 \\
0.50 \\
-3.32 \\
-3.28 \\
0.65 \\
4.86\end{array}$ & $\begin{array}{c}-0.80 \\
-7.29 \\
-19.23 \\
-13.38 \\
-7.07 \\
-10.55 \\
4.60\end{array}$ \\
\hline$n 100$ & $\begin{array}{l}0 \\
1 \\
2 \\
3 \\
4 \\
5 \\
6\end{array}$ & $\begin{array}{c}0.00 \\
-11.73 \\
-9.39 \\
10.39 \\
5.26 \\
3.59 \\
0.10\end{array}$ & $\begin{array}{c}0.00 \\
0.47 \\
0.49 \\
-1.21 \\
-0.91 \\
1.85 \\
-0.70\end{array}$ & $\begin{array}{c}0.00 \\
-0.18 \\
3.13 \\
1.85 \\
-1.04 \\
3.05 \\
2.98\end{array}$ & $\begin{array}{c}-0.81 \\
11.04 \\
-0.67 \\
7.72 \\
-14.80 \\
-10.80 \\
-20.70\end{array}$ \\
\hline$n 200$ & $\begin{array}{l}0 \\
1 \\
2 \\
3 \\
4 \\
5 \\
6\end{array}$ & $\begin{array}{c}0.00 \\
-4.61 \\
4.90 \\
18.19 \\
1.74 \\
1.15 \\
5.52\end{array}$ & $\begin{array}{c}0.00 \\
0.07 \\
1.84 \\
4.17 \\
-1.97 \\
-0.14 \\
5.19\end{array}$ & $\begin{array}{l}0.00 \\
6.52 \\
3.97 \\
2.77 \\
4.24 \\
5.54 \\
4.46\end{array}$ & $\begin{array}{c}-0.75 \\
-3.10 \\
-5.47 \\
-8.10 \\
-16.98 \\
-3.91 \\
-1.04\end{array}$ \\
\hline$n 300$ & $\begin{array}{l}0 \\
1 \\
2 \\
3 \\
4 \\
5 \\
6\end{array}$ & $\begin{array}{c}0.00 \\
-0.59 \\
-1.42 \\
4.09 \\
-4.92 \\
4.40 \\
5.35\end{array}$ & $\begin{array}{c}0.00 \\
3.33 \\
-1.04 \\
0.94 \\
-1.30 \\
1.33 \\
2.07\end{array}$ & $\begin{array}{c}0.00 \\
3.58 \\
-1.13 \\
0.39 \\
2.86 \\
1.72 \\
1.83 \\
\end{array}$ & $\begin{array}{c}0.10 \\
-0.73 \\
-3.77 \\
-5.68 \\
-19.77 \\
2.81 \\
-0.85\end{array}$ \\
\hline Average & - & +0.95 & +0.67 & +1.97 & -5.37 \\
\hline
\end{tabular}

The results are shown in Table III. For each dataset, we performed voltage island driven floorplanning with the number of voltage islands generated ranges from zero to six. ${ }^{11} \mathrm{We}$ could see from the results that up to $50 \%$ power saving can be achieved without any significant degradation in area and wire length. In addition, the speed is very acceptable and promising. Some resultant floorplans are shown in Figs. 3 and 4. Fig. 3 is a resultant packing of dataset $n 100$, with four voltage islands generated. In Fig. 4, we aim at testing a particular situation in which some cores can be operated at very low voltages. We use playout of the MCNC benchmark as the test case, and assign $0.6-\mathrm{V}$ to cores $\{2-9\}$, and $0.8-\mathrm{V}$ to cores $\{12-19\}$, respectively, as their minimum working voltage. Floorplanning

\footnotetext{
${ }^{11}$ Although the number of voltage levels is five, the number of islands is not limited by five because two islands can be operated at the same voltage.
} 
TABLE VI

EXPERIMENTAL RESUltS FOR LOWEST POSSIBLE POWER APPROACH

\begin{tabular}{|l|c|c|c|c|c|c|c|c|}
\hline Data & $K$ & $\begin{array}{c}\text { Total } \\
\text { Power }\end{array}$ & $\begin{array}{c}\text { Power } \\
\text { Saving } \\
(\%)\end{array}$ & $\begin{array}{c}\text { Total } \\
\text { Area }\end{array}$ & $\begin{array}{c}\text { Dead } \\
\text { Space } \\
(\%)\end{array}$ & $\begin{array}{c}\text { Wire } \\
\text { Length } \\
(\times 10)\end{array}$ & $\begin{array}{c}\text { No. of } \\
\text { Idle } \\
\text { Islands }\end{array}$ & $\begin{array}{c}\text { Run } \\
\text { Time } \\
(\mathrm{s})\end{array}$ \\
\hline$n 10$ & 4 & 267263 & 46.416 & 225032 & 1.490 & 1535 & 1 & 3.30 \\
\hline$n 30$ & 8 & 213796 & 54.446 & 221016 & 5.622 & 4012 & 2 & 39.06 \\
\hline$n 50$ & 7 & 191360 & 57.171 & 221552 & 10.369 & 8745 & 4 & 105.75 \\
\hline$n 100$ & 20 & 203397 & 49.639 & 185450 & 3.208 & 15778 & 0 & 326.30 \\
\hline$n 200$ & 29 & 199201 & 49.609 & 178723 & 1.693 & 32647 & 7 & 1416.87 \\
\hline$n 300$ & 30 & 289226 & 52.943 & 298419 & 8.461 & 60022 & 2 & 2723.68 \\
\hline
\end{tabular}

is then performed with $K=2$ and $1.5-\mathrm{V}$ being the chip-level voltage. In the result, two islands are generated as expected, one with cores $\{2,3,4,8,9\}$ and the other with $\{12,13,14$, $15,17\}$. The cores $\{5,6,7\}$ and $\{16,18,19\}$ are not included in the islands due to other factors like interconnect and area (their sizes are small and will not cause large power wastage even if operated at a higher voltage).

\section{A. Comparison With Previous Work}

In order to compare with the results of the previous related work [7] on exactly the same problem, ${ }^{12}$ we have done another set of experiments with the benchmarks provided by the authors of [7] without idle islands. In their datasets, the available voltage levels for each cell are chosen from the set $\{1.1-V, 1.3-V, 1.5-V, 1.8-V\}$, the area of each level shifter is $10 \times 10$ and the power cost is one. The comparisons are displayed in Table II. Results show that our approach is much more efficient and is able to save more power in most cases, while with less area overhead. Note that we set $K=4$ in the experiments, i.e., four islands are generated for each test case.

\section{B. Comparison With the Simplified Dynamic Programming Approach}

In this simplified dynamic programming approach, the islands formed across the left and right subtrees are not taken into account, This approach is suboptimal in terms of power saving with respect to a particular candidate floorplan, but due to the stochastic property of the annealing process, the solution quality will not be significantly degraded. This observation is supported by the experiments. Table IV displays the experimental results of this simplified dynamic programming approach, while Table V compares this approach with our approach, from which we can see that this simplified dynamic programming approach performs a little bit inferior to our method in terms of solution quality, with a $5.37 \%$ reduction in running time on average.

\section{Comparison With the Lowest Possible Power Approach}

In this lowest possible power approach, each core is set to operate at its lowest legal working voltage level, so that the largest possible power saving can be achieved. This approach provides an upper bound on power reduction for us

\footnotetext{
${ }^{12}$ In [7], it is also assumed that the legal voltage levels of a core are the feasible operating voltages at which the core will satisfy its performance requirement.
}

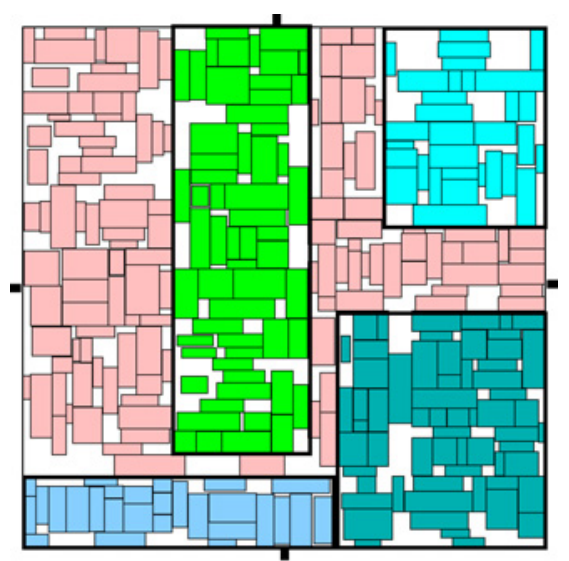

(a)

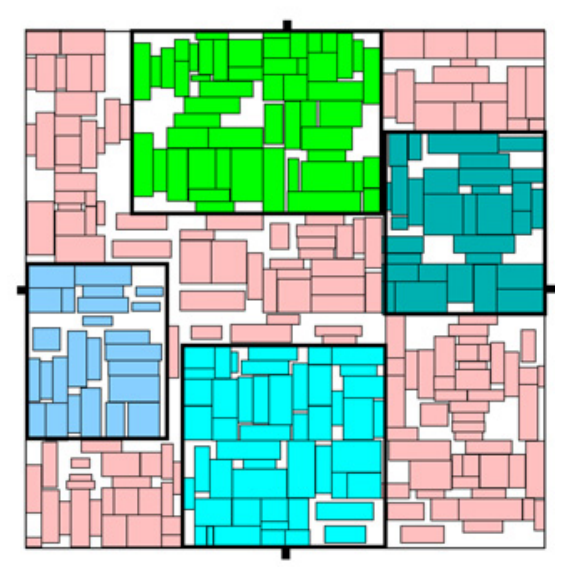

(b)

Fig. 5. Resultant floorplans for proximity constraints to power pins. (a) Without considering proximity to power pins. (b) Considering proximity to power pins.

to compare with. From Table III, we can see that the power consumption of our approach is very close to this lowest possible value, but the number of voltage islands is much smaller. Note that the efficient implementation of the island counting procedure [pseudocode IslandCount( $)$ ] contributes to the relatively shorter execution time of this lowest possible power approach.

\section{Extension to Consider Area and Power Overhead Due to Level Shifters}

Level shifters (LS) are needed for connections between two blocks in different power domains. These level shifters will 
TABLE VII

CONSIDERING THE POWER AND AREA OVERHEAD OF LEVEL SHIFTERS

\begin{tabular}{|l|c|c|c|c|c|c|c|c|}
\hline \multirow{3}{*}{ Data } & \multicolumn{2}{|c|}{ No. of LS } & Power Saving (\%) & \multicolumn{2}{c|}{ Area (Dead Space) } & \multicolumn{2}{c|}{ Run Time (s) } \\
\cline { 2 - 9 } & $\begin{array}{c}\text { w/o LS } \\
\text { Opt. }\end{array}$ & $\begin{array}{c}\text { With LS } \\
\text { Opt. }\end{array}$ & $\begin{array}{c}\text { w/o LS } \\
\text { Opt. }\end{array}$ & $\begin{array}{c}\text { With LS } \\
\text { Opt. }\end{array}$ & $\begin{array}{c}\text { w/o LS } \\
\text { Opt. }\end{array}$ & $\begin{array}{c}\text { With LS } \\
\text { Opt. }\end{array}$ & $\begin{array}{c}\text { w/o LS } \\
\text { Opt. }\end{array}$ & $\begin{array}{c}\text { With LS } \\
\text { Opt. }\end{array}$ \\
\hline$n 10$ & 34 & 15 & 12.42 & 17.83 & $300394(26.20)$ & $256836(13.68)$ & 0.667 & 1.095 \\
\hline$n 30$ & 38 & 38 & 40.07 & 40.41 & $236410(11.76)$ & $236222(11.70)$ & 8.076 & 17.28 \\
\hline$n 50$ & 103 & 73 & 28.95 & 36.58 & $242586(18.14)$ & $228655(13.15)$ & 23.94 & 63.71 \\
\hline$n 100$ & 207 & 167 & 19.45 & 24.39 & $219795(18.33)$ & $212630(15.56)$ & 88.05 & 303.4 \\
\hline$n 200$ & 372 & 316 & 15.93 & 16.94 & $218822(19.71)$ & $209078(15.96)$ & 378.2 & 1641 \\
\hline$n 300$ & 665 & 460 & 10.95 & 11.53 & $346578(21.18)$ & $308149(11.35)$ & 927.6 & 3321 \\
\hline Average & 236.5 & 178.1 & 21.29 & 24.61 & $260764(19.22)$ & $241928(13.56)$ & 237.7 & 891.2 \\
\hline Diff(\%) & \multicolumn{3}{|c|}{-24.7} & \multicolumn{3}{|c|}{+15.61} & \multicolumn{3}{|c|}{-7.22} & \multicolumn{3}{c|}{+275} \\
\hline
\end{tabular}

TABLE VIII

EXPERIMENTAL RESULTS FOR MINVDD APPROACH

\begin{tabular}{|c|c|c|c|c|c|c|c|c|c|c|c|c|c|c|c|}
\hline Data & $K$ & $\begin{array}{l}\text { Total } \\
\text { Power }\end{array}$ & $\begin{array}{c}\text { Power } \\
\text { Saving } \\
(\%)\end{array}$ & $\begin{array}{c}\text { Dead } \\
\text { Space } \\
(\%)\end{array}$ & $\begin{array}{c}\text { Wire } \\
\text { Length } \\
(\times 10)\end{array}$ & $\begin{array}{l}\text { No. of } \\
\text { Idle } \\
\text { Islands }\end{array}$ & $\begin{array}{l}\text { Run } \\
\text { Time } \\
\text { (s) }\end{array}$ & Data & $K$ & $\begin{array}{c}\text { Total } \\
\text { Power }\end{array}$ & $\begin{array}{c}\text { Power } \\
\text { Saving } \\
(\%)\end{array}$ & $\begin{array}{c}\text { Dead } \\
\text { Space } \\
(\%)\end{array}$ & $\begin{array}{c}\text { Wire } \\
\text { Length } \\
(\times 10)\end{array}$ & $\begin{array}{l}\text { No. of } \\
\text { Idle } \\
\text { Islands }\end{array}$ & $\begin{array}{l}\text { Run } \\
\text { Time } \\
\text { (s) }\end{array}$ \\
\hline$n 10$ & $\begin{array}{l}1 \\
2 \\
3 \\
4\end{array}$ & $\begin{array}{l}498778 \\
334549 \\
290593 \\
277862 \\
272102 \\
267263 \\
267263\end{array}$ & $\begin{array}{l}0.000 \\
32.92 \\
41.74 \\
44.29 \\
45.45 \\
46.41 \\
46.41\end{array}$ & $\begin{array}{l}1.158 \\
4.039 \\
0.548 \\
0.733 \\
0.638 \\
0.426 \\
0.460 \\
\end{array}$ & $\begin{array}{l}1274 \\
1293 \\
1463 \\
1438 \\
1340 \\
1359 \\
1320 \\
\end{array}$ & $\begin{array}{l}0 \\
0 \\
1 \\
1 \\
1 \\
2 \\
2 \\
\end{array}$ & $\begin{array}{l}0.491 \\
0.532 \\
0.784 \\
0.683 \\
0.824 \\
0.956 \\
0.973 \\
\end{array}$ & $n 100$ & $\begin{array}{l}0 \\
1 \\
2 \\
3 \\
4 \\
5 \\
6\end{array}$ & $\begin{array}{l}403877 \\
286710 \\
264563 \\
248192 \\
244842 \\
237291 \\
227722\end{array}$ & $\begin{array}{l}0.000 \\
29.01 \\
34.49 \\
38.55 \\
39.38 \\
41.24 \\
43.62 \\
\end{array}$ & $\begin{array}{l}1.052 \\
6.767 \\
8.728 \\
3.791 \\
1.918 \\
6.848 \\
0.891 \\
\end{array}$ & $\begin{array}{l}12536 \\
13294 \\
13222 \\
14205 \\
13957 \\
14138 \\
13725 \\
\end{array}$ & $\begin{array}{l}0 \\
0 \\
0 \\
0 \\
0 \\
0 \\
0 \\
\end{array}$ & $\begin{array}{l}85.18 \\
85.85 \\
88.73 \\
90.61 \\
95.08 \\
99.03 \\
103.7 \\
\end{array}$ \\
\hline$n 30$ & $\begin{array}{l}1 \\
2 \\
3 \\
4 \\
5 \\
6\end{array}$ & $\begin{array}{l}469330 \\
275553 \\
271589 \\
221819 \\
221819 \\
212608 \\
209293 \\
\end{array}$ & $\begin{array}{l}0.000 \\
41.28 \\
42.13 \\
52.74 \\
52.74 \\
54.70 \\
55.41 \\
\end{array}$ & $\begin{array}{l}1.476 \\
0.438 \\
0.874 \\
3.082 \\
0.720 \\
0.698 \\
0.787 \\
\end{array}$ & $\begin{array}{l}3420 \\
3707 \\
3496 \\
3592 \\
3457 \\
3521 \\
3754 \\
\end{array}$ & $\begin{array}{l}0 \\
0 \\
0 \\
1 \\
2 \\
2 \\
3 \\
\end{array}$ & $\begin{array}{l}7.014 \\
7.169 \\
8.307 \\
8.539 \\
8.919 \\
9.632 \\
10.24 \\
\end{array}$ & $n 200$ & $\begin{array}{l}4 \\
5 \\
6\end{array}$ & $\begin{array}{l}395316 \\
329242 \\
291271 \\
286235 \\
269032 \\
259551 \\
254794\end{array}$ & $\begin{array}{l}0.000 \\
16.71 \\
26.32 \\
27.59 \\
31.94 \\
34.34 \\
35.54 \\
\end{array}$ & $\begin{array}{l}6.219 \\
2.398 \\
3.753 \\
4.943 \\
0.892 \\
4.124 \\
1.017 \\
\end{array}$ & $\begin{array}{l}31893 \\
30558 \\
30031 \\
30729 \\
30412 \\
31387 \\
30706 \\
\end{array}$ & $\begin{array}{l}0 \\
0 \\
0 \\
0 \\
0 \\
0 \\
0 \\
\end{array}$ & $\begin{array}{l}364.9 \\
383.6 \\
394.4 \\
405.2 \\
428.3 \\
460.8 \\
487.1 \\
\end{array}$ \\
\hline$n 50$ & $\begin{array}{l}0 \\
1 \\
2 \\
3 \\
4 \\
5 \\
6\end{array}$ & $\begin{array}{l}446803 \\
285954 \\
248255 \\
231630 \\
204658 \\
191360 \\
191360 \\
\end{array}$ & $\begin{array}{l}0.000 \\
36.00 \\
44.43 \\
48.16 \\
54.19 \\
57.17 \\
57.17\end{array}$ & $\begin{array}{l}0.606 \\
0.715 \\
0.807 \\
2.058 \\
0.775 \\
1.124 \\
0.764\end{array}$ & $\begin{array}{l}7765 \\
8004 \\
7866 \\
7826 \\
8097 \\
8003 \\
8815 \\
\end{array}$ & $\begin{array}{l}0 \\
0 \\
0 \\
0 \\
1 \\
2 \\
3 \\
\end{array}$ & $\begin{array}{l}20.11 \\
21.19 \\
22.15 \\
23.46 \\
24.66 \\
25.41 \\
26.87\end{array}$ & $n 300$ & $\begin{array}{l}4 \\
5 \\
6\end{array}$ & $\begin{array}{l}614632 \\
393365 \\
379667 \\
361630 \\
353570 \\
350212 \\
342367\end{array}$ & $\begin{array}{l}0.000 \\
36.00 \\
38.23 \\
41.16 \\
42.47 \\
43.02 \\
44.30\end{array}$ & $\begin{array}{l}2.594 \\
4.814 \\
1.845 \\
2.879 \\
1.175 \\
6.196 \\
3.432 \\
\end{array}$ & $\begin{array}{l}51507 \\
49823 \\
47248 \\
53672 \\
48355 \\
50268 \\
50773\end{array}$ & $\begin{array}{l}0 \\
0 \\
0 \\
0 \\
0 \\
0 \\
0\end{array}$ & $\begin{array}{l}742.9 \\
793.8 \\
802.3 \\
812.7 \\
855.6 \\
866.1 \\
912.4 \\
\end{array}$ \\
\hline
\end{tabular}

lead to area and power overhead and should be minimized. We can extend our floorplanner to minimize the usage of level shifters by having additional terms in the cost function that represents the total area and power consumption due to level shifters. We assume that a level shifter is needed whenever a wire is connecting two blocks operating at different voltages. For example, if a net connects a source in voltage island $A$ to three sinks, two in island $B$ and one in island $C$, two level shifters will be inserted, one between $A$ and $B$ and one between $A$ and $C$. These level shifters will add to the total area and power consumption of the design. This extra area and power usage can be estimated according to the size of a level shifter which can then be included into the area and power term of the cost function, $\psi_{\mathrm{LS}}=\left(A+A_{\mathrm{LS}}\right)+\lambda_{w} W+\lambda_{p}\left(P+P_{\mathrm{LS}}\right)$, where $A_{\mathrm{LS}}$ and $P_{\mathrm{LS}}$ are the total area and power consumption due to the level shifters, respectively. ${ }^{13}$ The result is shown

\footnotetext{
${ }^{13}$ The extra area due to the level shifters are considered and reflected in the cost function as described above. In terms of the final layout, we can proportionally increase the area of each core according to the number of level shifters inserted due to signals coming out from it. In this way, the final floorplan obtained from the annealing process can be slightly changed to take into account this extra area due to the level shifters.
}

in Table VII. In these experiments, the number of voltage islands $K$ is set to four. Results show that our method can reduce the number of level shifters by $67.1 \%$ on average and can reduce total power consumption as well as total area, with some penalty in run time.

\section{E. Extension to Consider Power Network Routing}

The voltage islands should be placed in proximity to the power pins to simplify the power routing step and to minimize the $I R$ drop. The power network resources can be modeled by the sum of the half perimeters of the islands [5]. We can also extend our floorplanner to consider these power network issues by having additional terms in the cost function (with weights determined by random walk) to represent: 1) the total distance of the voltage islands from their respective power pins; and 2) the sum of the half perimeters of the islands. In our experiments, we assume that the positions of the $K$ power pins are given. In each iteration of the annealing process, each island is matched to a power pin such that the total distance between them is the smallest possible. This total distance and the sum of the islands' half perimeters will be minimized during the annealing process. 
Two resultant packings of the $n 300$ dataset consisting of 300 blocks are shown in Fig. 5, and they are produced with the fixed-outline constraint. The packing in Fig. 5(a) is obtained by the original floorplaner, without taking into consideration these power network issues, while the one in Fig. 5(b) is obtained by this extended version. There are four power pins located at the center of each boundary in this example. We can see from the figures that the four islands are shifted to the sides of the chip containing the pins in order to be located closer to their respective power pins, and the islands are closer to square in shape that will favor $I R$ drop reduction and power network routing.

\section{F. Experiments With minVdd}

In this version of the problem, instead of having a few discrete legal voltage levels, each core has a minimum voltage $\min V_{i}$ such that $i$ can achieve timing closure at a voltage level not less than $\min V_{i}$. Our framework can be easily modified to address this minVdd version of the problem. The results of applying our dynamic programming approach to this problem is shown in Table VIII. We can see that the running time is much shorter in comparison with Table III because it is easier to compute the maximum $\min V_{i}$ among a group of cores than finding the smallest voltage level feasible to all of them.

\section{CONCLUSION}

In this paper, we have proposed a simulated annealing-based approach for the floorplanning problem with simultaneous island partitioning and voltage assignment. The three factors area, wire length and power consumption of the resultant floorplan are concurrently taken into consideration, and the experiment results have shown that we are able to achieve a significant power saving of up to $50 \%$ for the testing datasets.

In addition, when extended to minimize the number of level shifters between different voltage domains, our method can reduce $67.1 \%$ of the LS used on average, with some penalty in power saving; and it also functions well to generate voltage islands in proximity to their corresponding power pins, by having an additional term in the cost function to minimize the total distance between voltage islands and power pins.

\section{REFERENCES}

[1] Y. Cai, B. Liu, Q. Zhou, and X. Hong, "Voltage island generation in cell-based dual-vdd design," Inst. Electron., Informat. Commun. Eng. Trans. Fundam. Electron., Commun. Comput. Sci., vol. E90-A, no. 1, pp. 267-273, 2007.
[2] L. Guo, Y. Cai, Q. Zhou, and X. Hong, "Logic and layout aware voltage island generation for low power design," in Proc. Asian South Pacific Design Automat. Conf., 2007, pp. 666-671.

[3] J. Hu, Y. Shin, N. Dhanwada, and R. Marculescu, "Architecting voltage islands in core-based system-on-a-chip designs," in Proc. Int. Symp. Low Power Electron. Design, 2004, pp. 180-185.

[4] W.-L. Hung, G. M. Link, Y. Xie, N. Vijaykrishnan, N. Dhanwada, and J. Conner, "Temperature-aware voltage islands architecting in systemon-chip design," in Proc. Comput. Design, 2004, pp. 689-696.

[5] W.-P. Lee, H.-Y. Liu, and Y.-W. Chang, "Voltage island aware floorplanning for power and timing optimization," in Proc. Int. Conf. Comput.Aided Design, 2006, pp. 389-394.

[6] Q. Ma and E. F. Y. Young, "Voltage island-driven floorplanning," in Proc. Int. Conf. Comput.-Aided Design, 2007, pp. 644-649.

[7] W.-K. Mak and J. W. Chen, "Voltage island generation under performance requirement for SoC designs," in Proc. Asian South Pacific Design Automat. Conf., 2007, pp. 798-803.

[8] D. Sengupta and R. Saleh, "Application-driven floorplan-aware voltage island design," in Proc. 45th Assoc. Comput. Machinery/IEEE Design Automat. Conf., 2008, pp. 155-160.

[9] X. Tang, R. Tian, and D. F. Wong, "Minimizing wire length in floorplanning," IEEE Trans. Comput.-Aided Design Integrat. Circuits Syst., vol. 25 , no. 9 , pp. $1744-1753$, Sep. 2006.

[10] D. F. Wong and C. L. Liu, "A new algorithm for floorplan design," in Proc. 23rd Assoc. Comput. Machinery/IEEE Design Automat. Conf., 1986, pp. 101-107.

[11] H. Wu, I.-M. Liu, D. F. Wong, and Y. Wang. "Postplacement voltage island generation under performance requirement," in Proc. Int. Conf. Comput.-Aided Design, 2005, pp. 309-316.

[12] S. Yang, W. Wolf, N. Vijaykrishnan, and Y. Xie. "Reliability-aware SoC voltage islands partition and floorplan," in Proc. Emerging Very Large Scale Integrat. Technol. Architect., 2006, p. 343.

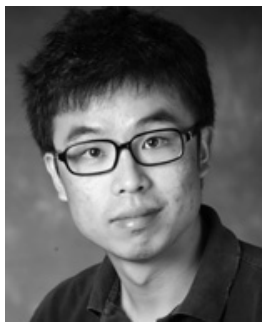

Qiang Ma received the B.Eng. degree in electrical engineering from Zhejiang University, Hangzhou, China, in 2006, and the M.Phil. degree in computer science from the Chinese University of Hong Kong, Shatin, Hong Kong, China, in 2008. He is currently working toward the Ph.D. degree at the Department of Electrical and Computer Engineering, University of Illinois at Urbana-Champaign, Champaign.

His research interests include physical design of chips, packages, and printed circuit boards.

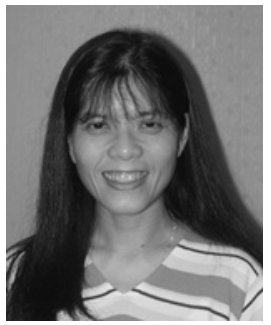

Evangeline F. Y. Young received the B.S. and M.Phil. degrees in computer science from the Chinese University of Hong Kong (CUHK), Hong Kong, China, and the Ph.D. degree in computer science from the University of Texas, Austin, in 1999.

From 1999 to 2004, she was as an Assistant Professor with the Department of Computer Science and Engineering, CUHK, and currently she is an Associate Professor in the same department. Her current research interests include algorithms and computer aided design of very large scale integration circuits. She is now working actively on floorplanning, placement, routing, and algorithmic designs.

Dr. Young has served on the technical program committees of several major conferences including the International Conference on Computer Aided Design, the Asia and South Pacific Design Automation Conference, the International Symposium on Physical Design, and the Great Lakes Symposium Very Large Scale Integration. 